\title{
A Rapid and Precise On-Response in Posterior Parietal Cortex
}

\author{
James W. Bisley, ${ }^{1}$ B. Suresh Krishna, ${ }^{1}$ and Michael E. Goldberg ${ }^{1,2}$ \\ ${ }^{1}$ Mahoney Center for Brain and Behavior, Center for Neurobiology and Behavior, Columbia University College of Physicians and Surgeons, and the New \\ York State Psychiatric Institute, New York, New York 10032, and ²Departments of Neurology and Psychiatry, Columbia University College of Physicians and \\ Surgeons, New York, New York 10032
}

The activity of neurons in the lateral intraparietal area (LIP) of the monkey predicts the monkey's allocation of spatial attention. We show here that despite being relatively high within the visual hierarchy, neurons in LIP have extremely short and precise visual latencies. Mean latency was $45.2 \mathrm{msec}$; the timing precision of the onset response was usually better than $4 \mathrm{msec}$. The majority of neurons had a pause in response after an initial burst, followed by more sustained visual activity. Previous attention allocation had no effect on either the latency or magnitude of the initial burst, but produced clear effects on the magnitude of the later sustained activity. Together, these data indicate that the initial burst in LIP visual response reflects an uncontaminated sensory signal. Information about stimulus onset is transmitted rapidly through the visual system to LIP; the on-response has a higher speed and temporal precision than realized previously. This information could be used to orient attention to novel objects in the visual environment rapidly and reliably.

Key words: neural coding; reliability; attention; parietal cortex; spike timing; vision

\section{Introduction}

The lateral intraparietal area (LIP) is a region of posterior parietal cortex with connections to both higher-order visual areas and the oculomotor system (Andersen et al., 1990). Human psychophysical studies have shown that attention can be automatically attracted to the location of a suddenly appearing stimulus (Yantis and Jonides, 1984; Egeth and Yantis, 1997) or to the location of a planned saccade (Shepherd et al., 1986; Hoffman and Subramaniam, 1995). We have shown recently that this is also true in the monkey and that the activity in LIP predicts the locus of attention on a moment-by-moment basis, whether it be drawn by a sudden visual onset or a saccade plan (Bisley and Goldberg, 2003a). Neurons in early parts of the visual system, including the retina (Brivanlou et al., 1998), thalamus (Reinagel and Reid, 2000; Liu et al., 2001), striate cortex (Victor and Purpura, 1996), and the middle temporal (MT) area (Buracas et al., 1998; Bair et al., 2002) respond with precisely timed spikes to the onset of a stimulus. A similarly fast and precisely timed response in LIP could subserve the rapid and reliable allocation of attention to new stimuli in the visual environment. Therefore, this study examined the initial visual response in LIP to sudden onsets to determine whether there is a precisely timed signal similar to that seen earlier in the visual system.

Received Nov. 7, 2003; revised Dec. 17, 2003; accepted Dec. 22, 2003.

This work was supported by the National Eye Institute, the Human Frontiers Science Project, the James $S$. MacDonnell Foundation, the W. M. Keck Foundation, and the Whitehall Foundation. We thank the members of the Mahoney Center for Brain and Behavior for comments on this manuscript.

Correspondence should be addressed to Dr. James W. Bisley, Center for Neurobiology and Behavior, Columbia University, 1051 Riverside Drive, Unit 87, Kolb Research Annex, Room 5-06, New York, NY 10032. E-mail: jwb2003@columbia.edu.

DOI:10.1523/JNEUROSCI.5007-03.2004

Copyright $\odot 2004$ Society for Neuroscience $\quad$ 0270-6474/04/241833-06\$15.00/0

\section{Materials and Methods}

Subjects. All experimental protocols were approved by the National Eye Institute Animal Care and Use Committee as complying with the guidelines established in the Public Health Service Guide for the Care and Use of Laboratory Animals. Two male rhesus monkeys (Macaca mulatta) had scleral search coils, head restraining devices, and recording chambers implanted during sterile surgery under ketamine and isoflurane anesthesia. Chambers were placed using magnetic resonance images, and neurons were identified as being in LIP by their consistent visual, delayperiod, and saccade-related responses in a memory-guided saccade task, as well as by the location of the intraparietal sulcus within the chamber according to the images. Data were collected from neurons that had a strong response while the stimulus was present (visual activity), independent of whether they had activity during the delay or perisaccadic periods of a memory-guided saccade.

Stimuli and behavioral task. Behavioral control and data collection were done on computers using the REX system (Hays et al., 1982). Visual stimuli were back-projected on a tangent screen by a Multisync LT100 DLP projector (NEC, Tokyo, Japan) calibrated with a Tektronix (Wilsonville, OR) J17 Photometer. The background luminance was $15 \mathrm{~cd} / \mathrm{m}^{2}$ and the projector refresh rate was $60 \mathrm{~Hz}$. Stimulus timing was calculated by measuring a pulse from a photocell affixed to the back of the screen and illuminated by a small square on the corner of the same video frame as the appearance of any new stimulus. The monkey could not see the photocell or its illumination square.

The results reported here are an additional analysis of data collected in the Laboratory of Sensorimotor Research of the National Eye Institute, which were reported previously (Bisley and Goldberg, 2003a). Data were recorded while monkeys performed a variation of the memory-guided saccade task. Briefly, the monkey initiated a trial by fixating on a central spot. After a 1-2 sec delay, a second spot (the target; diameter, $0.2^{\circ}$; contrast, 55\%) appeared for $100 \mathrm{msec}$ either in the center of the receptive field (RF) or in the opposite quadrant. After the target was extinguished there was another delay of 800,1300 , or $1800 \mathrm{msec}$ and then four rings (thickness, $0.2^{\circ}$; diameter, $2.2^{\circ}$; contrast, $28 \%$ ) appeared for one video 
frame (17 msec), one in each quadrant at the possible target locations. One of the rings had a gap in it (the probe) and the monkey's task was to indicate the side of the probe that the gap was on by either making the planned saccade or maintaining fixation once the fixation spot was extinguished. The monkey received a reward of a drop of water for correctly indicating the orientation of the probe. The probe or a distracting ring was always presented in the RF. In $50 \%$ of the trials, a task-irrelevant distractor, identical to the target, was flashed for $100 \mathrm{msec}$ either at the target location or in the opposite quadrant $500 \mathrm{msec}$ after target offset.

Data collection and analysis. Single-unit activity was isolated with a Bak dual-window discriminator (BAK Electronics Inc., Germantown, MD) and recorded with $1 \mathrm{msec}$ precision by the REX software. Off-line analysis was performed on Matlab (MathWorks, Natick, MA). There were 90-300 (mean, 195) trials per neuron. Histograms with $2 \mathrm{msec}$ bins were aligned by stimulus onset, as indicated by the photocell. Response latency was calculated using a method similar to that described by Maunsell and Gibson (1992). For each stimulus, the distribution of the number of spikes (summed across all trials) in each of the 50 bins $(100 \mathrm{msec})$ before stimulus onset was fitted with a Poisson distribution. We verified the Poisson assumption in three ways. First, for all stimuli, the variance of the distribution of prestimulus activity was generally similar to the mean; few cells (one for target, two for ring) had values of the index-ofdispersion statistic (sum of squared deviations from the mean divided by the mean) that were significantly different from chance $\left(\chi^{2}\right.$ test; $p<0.01$; no correction for multiple comparisons). Consistent with this, the mean of the distribution of variance-to-mean ratios from all cells did not deviate significantly from one for either the target or the ring stimulus ( $t$ tests; $p>0.05$ ). Second, the Poisson fit explained at least $80 \%$ of the variance in $>85 \%$ of the cells; few cells (one for target, one for ring) showed significant deviations from the fit ( $\chi^{2}$ goodness-of-fit test; $p<$ 0.01; no correction for multiple comparisons). Finally, the sample average was almost identical to the parameter estimated from the Poisson fit (slope, 0.98; intercept, $0.10 ; R^{2}, 0.99$ for target; slope, 1.01; intercept, $0.08 ; R^{2}, 0.998$ for the ring).

To determine each latency, we first calculated a cutoff from the Poisson fit. The cutoff was the number of spikes below which the spike count would be expected to lie $99 \%$ of the time ( $p<0.01$ ). Response latency was defined as the time from stimulus onset to the time of the first of three consecutive poststimulus bins, each of which contained a number of spikes that was greater than or equal to the cutoff.

Bootstrap analysis. To assess the consistency of latency measurements, we ran a bootstrap analysis. For each cell we created a new data set by randomly sampling, with repetition, trials from the original data set until the new set had the same number of trials as the original. The latency was then calculated from this new data set. This was repeated 2000 times, giving a distribution of latencies.

\section{Results}

Data were analyzed from 41 LIP neurons (18 from monkey B; 23 from monkey I) with visual activity. Occasionally, neurons had insufficient trials to produce latencies in some stimulus conditions using the method described above (i.e., they did not have three consecutive bins with $p$ values $<0.01$ ). These neurons were excluded from analyses in those conditions; such cases are noted when relevant.

The responses of two typical neurons to the onset of a target or ring are plotted in Figure 1. They have an initial burst of activity, starting at a precise time, followed by a brief period of inactivity and then a period of more regular firing. It is important to note that the time scale of analysis here is small; thus, these response patterns all occur within the traditional visual response.

\section{Response latency}

There were nine stimulus configurations for which we measured latency. To examine the response to the target, all trials in which the target appeared in the RF could be used, because it was the first stimulus presented. To examine the response to the distractor appearing in the RF, trials were split into those in which the target had appeared in the RF and those in which the target had appeared opposite the RF. Finally, to examine the response to the rings, trials were split first into those in which the target had appeared in the RF or not and then by ring type: distractor ring, probe with the gap on the left, and probe with the gap on the right.

\section{Distribution of latencies in LIP}

Figure $2 \mathrm{~A}$ shows the cumulative distributions of response latencies from the target and solid ring for all 41 neurons. Three features stand out from this figure. First, the latencies were very short (means, $49.5 \mathrm{msec}$ for the target, $41.4 \mathrm{msec}$ for the ring). This was particularly true for the ring stimulus, for which over half of the neurons had latencies of $40 \mathrm{msec}$ or less. Second, the latencies to the target responses were slower than the latencies obtained from the ring. Third, the widths of the distributions were similar in the two cases, despite the latency difference. To determine whether the difference between the latencies was consistent among neurons, we plotted the target latency as a function of the ring latency and fitted a line to the data (Fig. $2 \mathrm{~B}$ ). We found 

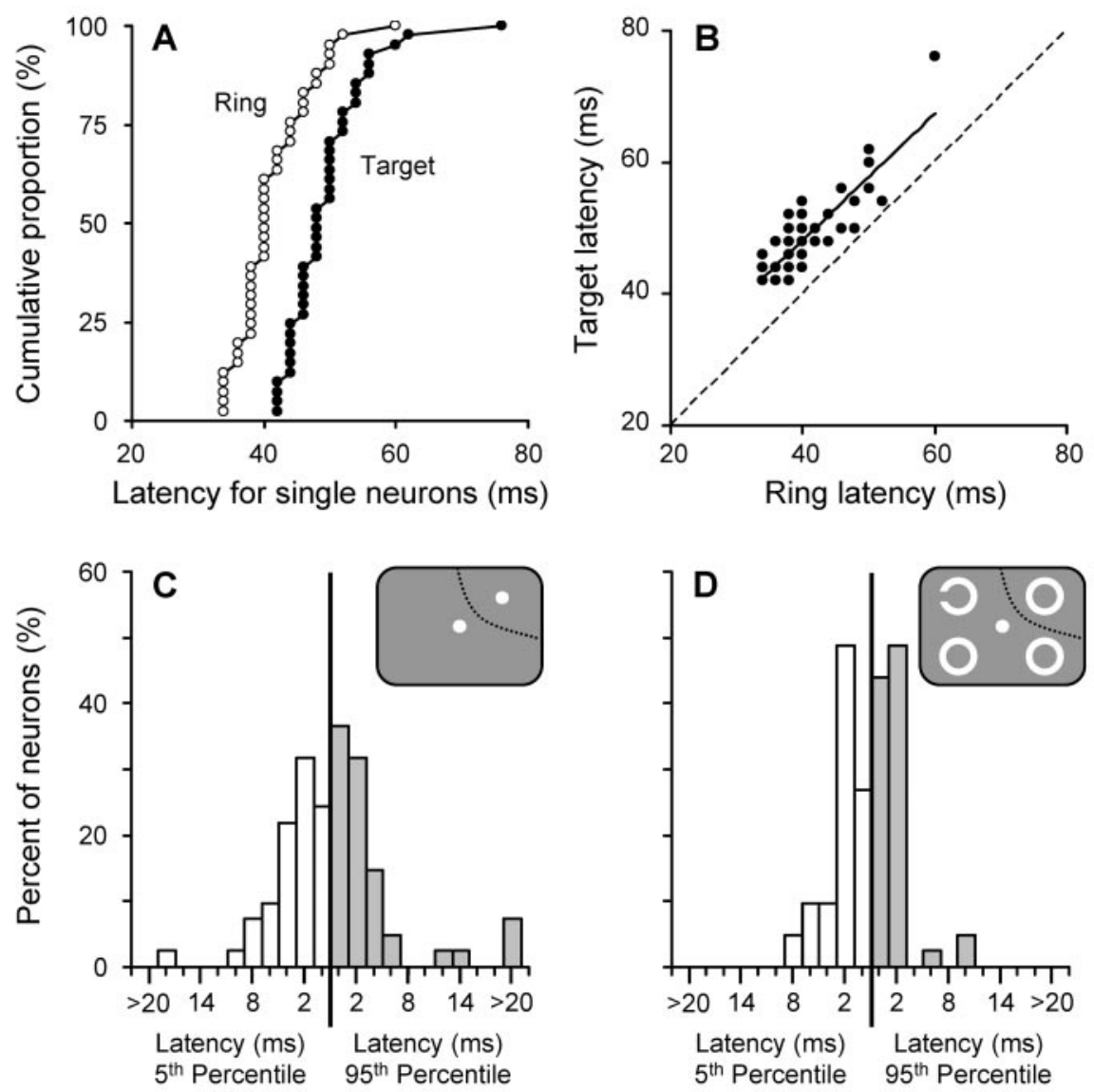

Figure 2. Latencies after the target and ring. $A$, Cumulative distribution of latencies after the presentation of the target and ring. $B$, Plotting target latency against ring latency shows a consistent relationship between the two. The linear best fit line is in black. C, D, Distributions of the 5 th (white columns) and 95th (gray columns) percentiles of latency after the target $(C$ and ring $(D)$.

a slope of 0.96 and an intercept of $9.78\left(R^{2}, 0.73 ; p \ll 0.01\right)$, suggesting that the target latency of each cell is shifted by $\sim 10$ msec relative to the ring latency. There was also a difference in the initial response (mean spike rate over the first $20 \mathrm{msec}$ of the response) to these stimuli (mean difference, 52.9 spikes/sec; $p \ll$ 0.001 ; paired $t$ test). This difference in response partially explains the difference in latency between the two stimuli $\left(R^{2}, 0.17 ; p<\right.$ $0.01)$. Similarly, there was a significant $\left(R^{2}, 0.18 ; p \ll 0.01\right)$ correlation between latency and initial visual response across all conditions.

\section{Latencies under different conditions}

We have shown previously that when a monkey is planning a memory-guided saccade, there is a region of enhanced contrast sensitivity at the goal of that saccade (Bisley and Goldberg, 2003a). To determine whether this attention enhancement had any effect on response latency, we measured the response latencies to the distractor and ring from trials in which a saccade was planned into or opposite the RF. In both cases, the mean latencies were not affected by the location of the target $(p>0.2$; paired $t$ test). Therefore, we have pooled these data for the remaining analyses.

\section{Latencies for similar stimuli}

Although the target and distractor were identical stimuli, they had differing behavioral relevance: the target was important for the task, the distractor was irrelevant. A comparison of the laten- cies to these stimuli showed a significant but marginal difference (mean difference, 0.8 msec; $p=0.03$; paired $t$ test; $n=37$ ).

One of three different rings could appear in the RF: the probe could appear in either orientation or the ring could appear without a gap. We have shown previously that there was a differential response to these stimuli 100-250 msec after they appear; however, no difference was seen in the response magnitude in the first 100 msec after stimulus onset (Bisley and Goldberg, 2003a). To test whether there was a difference in the timing of the initial response to these stimuli, we compared the latencies with the three stimuli. We found that the latencies for the two probes were similar ( $p>0.1$; paired $t$ test; $n=39$ ), but that the latencies for the complete ring were shorter than the latency to either of the probes (mean differences, $1.6 \mathrm{msec}$, $n=39 ; 2.2 \mathrm{msec}, n=40 ; p \ll 0.01)$.

\section{Consistency of latencies}

The neurons in Figure 1 showed a high degree of consistency in the timing of the initial response to a visual stimulus. To quantify this we ran a bootstrap analysis to find the distribution of latencies for each neuron. We found that the quartiles were the same as the median for most cells (63\% for the target; $81 \%$ for the ring) and were rarely $>2 \mathrm{msec}$ from the median (10\% for the target; $5 \%$ for the ring). Figure 2 shows the distribution of the distance of the 5th and 95 th percentiles from the median latencies for the target (Fig. 2C) and ring (Fig. $2 D$ ) stimuli. For the majority of neurons, the 5 th and 95 th percentiles lay within $4 \mathrm{msec}$ of the median for the target and within $2 \mathrm{msec}$ for the ring, confirming the consistency illustrated in Figure 1.

This result suggests that the first spike in the response occurs at a consistent time on each trial. To test this further, we measured the time of the first spike in each trial beginning at the latency for that neuron. As a control, a similar measurement was made of the time of the last spike before the latency. These data are plotted in Figure 3, which shows the responses to the target (Fig. $3 A$ ) and ring stimuli (Fig. 3B). In this figure, each row represents a single neuron and each column represents a $2 \mathrm{msec}$ bin radiating in either direction from the response latency. The brightness of each block represents the probability that the spike occurred in that bin, with darker blocks showing higher probability. The dots show the median time of last (prelatency) and first (postlatency) spike, and the cells have been sorted based on increasing median first spike time.

The most striking feature of these figures is that in over $75 \%$ of the neurons the median first spike time is within 6 msec for the target and $4 \mathrm{msec}$ for the ring, whereas the median last spike times are longer and more variable, indicative of the much lower firing rate before the onset of the visual response. This suggests that our calculation of latency is appropriate and that after the onset of a visual stimulus, most cells will fire a burst of activity starting at almost the same time on each trial. The last spike medians in 
Figure $3 B$ (left plot) are less than those in Figure $3 A$ (left plot) because of the higher prestimulus firing rate brought about by the frequent appearance of the target or distractor in the RF before the presentation of the rings.

Pattern of activity after a sudden onset

To examine the pattern of activity after stimulus onset in individual neurons more closely, we tested whether the response in that bin was greater than or equal to the cutoff for statistical significance calculated from the best-fitting Poisson distribution for the prestimulus activity (see Materials and Methods). These data are plotted in Figure 4, $A$ and $C$, with each row representing a single neuron and each column a $2 \mathrm{msec}$ bin starting at the latency for that neuron. Black blocks represent bins with significantly higher activity and white blocks represent bins with activity that was not significantly higher.

The example neurons in Figure 1 show that initially there is a rapid burst of activity, after which there was a pause in response, followed by more sustained visual activity. Such an initial burst, defined as significantly greater activity (at least 20\% greater and statistically significant) in the first $20 \mathrm{msec}$ of the response compared with the activity 50-70 msec into the response, was found in 34 of 41 neurons $(82.9 \%)$; it lasted for $8-26 \mathrm{msec}$ (mean, 15.4 and $17.8 \mathrm{msec}$ for target and ring stimuli, respectively). Previous studies have shown that the net visual response to a distractor appearing in the same place as the target is smaller than the response to the distractor appearing previously (Powell and Goldberg, 2000). Here, we found that during the initial burst this difference was not present $(p>0.9$; paired $t$ test $)$ (Fig. $4 B$ ). The lack of effect is not the result of response saturation attributable to the higher firing rates in the on-response, because the same neurons showed much higher firing rates in response to the ring over the same-onset time period (see above). However, during the sustained visual response, the activity to the distractor appearing at the target site was significantly less than the activity to the distractor appearing previously $(p<0.01)$ (Fig. $4 D$ ).

Neurons were considered to have a period of inactivity if two criteria were met: (1) Between $10 \mathrm{msec}$ and $50 \mathrm{msec}$ after the latency (Fig. $4 A, C$, period between vertical lines), at least two bins ( $4 \mathrm{msec}$ ) had to have activity that was not significantly higher than the prestimulus activity. (2) In the period between 50 and $100 \mathrm{msec}$ after the latency, at least 10 bins ( $20 \mathrm{msec}$ ) had to show significantly higher activity. This second criterion ruled out neurons with only an

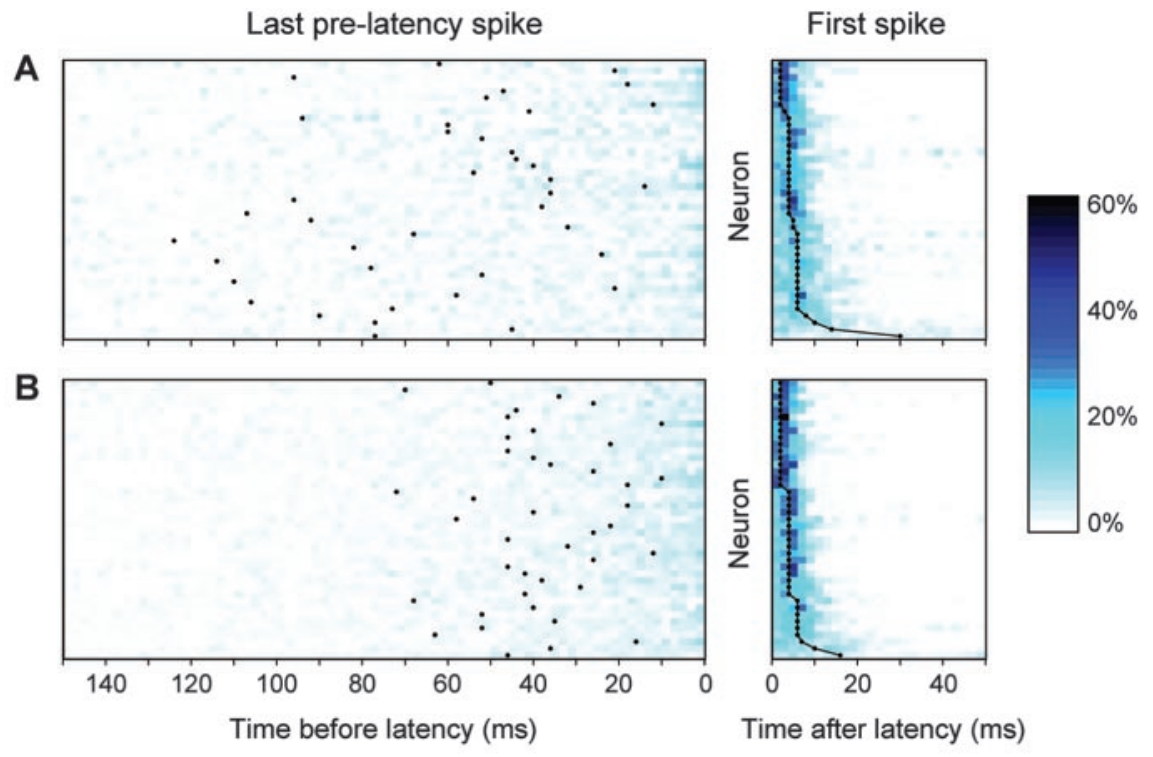

Figure 3. Distribution of last prelatency (left plots) and first postlatency (right plots) spike probabilities for the target $(A)$ and ring $(B)$. Each row represents a single neuron. Brightness represents the probability of a spike occurring in a given bin; darker blocks show higher probabilities. Dots show the median time of the last (left plots) and first (right plots) spikes. Neurons were sorted by the median time of the first spike.
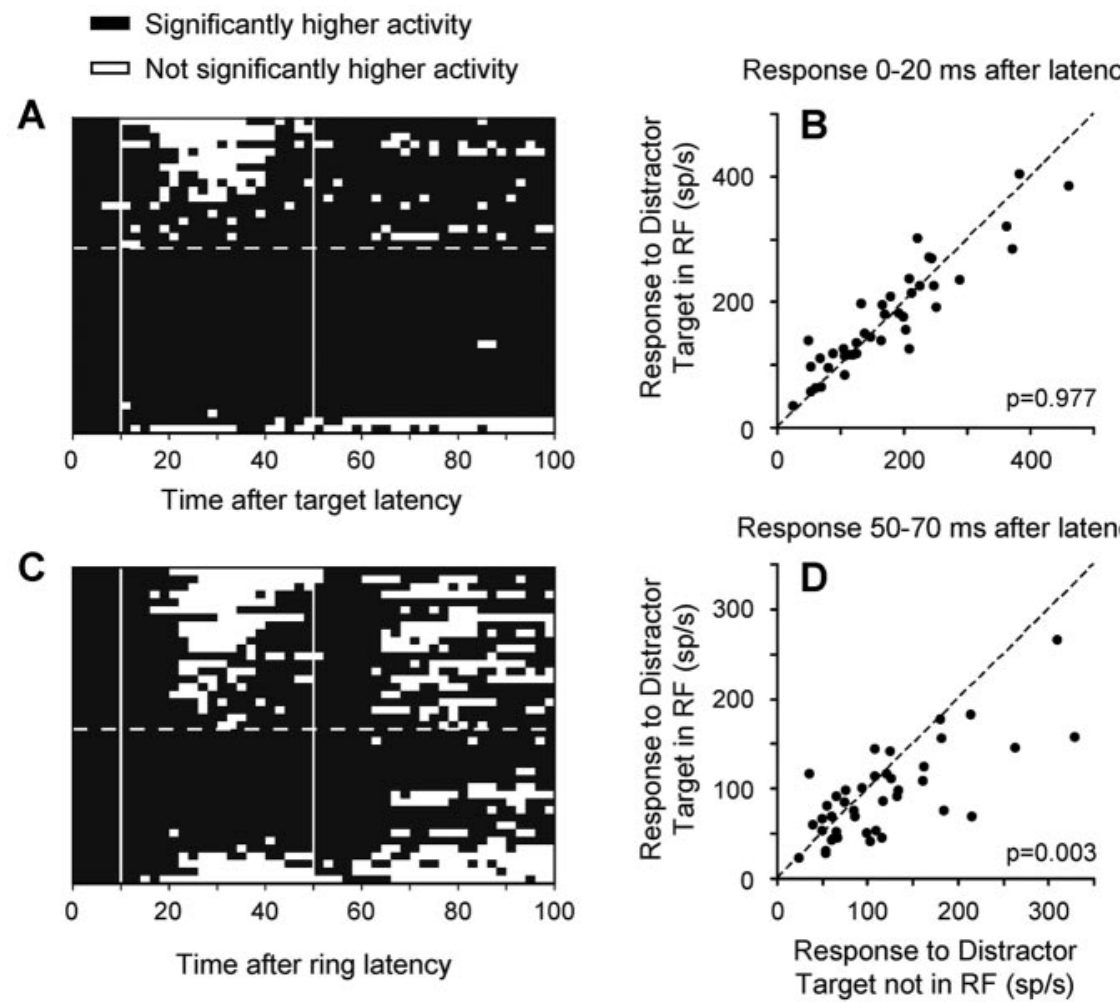

Figure 4. Postlatency activity. $A, C$, Distribution of activity that was (black) or was not (white) significantly higher than the prestimulus activity for all 41 cells after the target $(A)$ or ring $(C)$. Each row shows data from a single cell. Cells were sorted by whether they had a period of inactivity (above the dashed lines) or not, and then by the number of white blocks. The vertical white lines are at 10 and $50 \mathrm{msec}$ (see text). $B, D$, Distractor responses during the initial burst $(B)$ or $50-70$ msec postlatency $(D)$. Data from trials in which the target preceded the distractor in the RF plotted against data from trials in which the target did not appear in the RF. $p$ values from paired $t$ tests.

initial on-response but no maintained activity. The horizontal dashed line indicates the border between neurons that passed these criteria (above the dashed line) and those that failed. Neurons were more likely to have a period of inactivity after the 
presentation of a ring (51.2\%) than after the target (41.5\%). After the ring there was no correlation between the strength of the initial response and the presence or absence of a period of inactivity (linear regression of visual response as a function of inactivity; $p>0.1$ ). However, there was a slight, but significant correlation between the response and presence of inactivity after the target: neurons with higher responses were more likely to have a period of inactivity $\left(R^{2}, 0.21 ; p<0.01\right)$.

\section{Discussion}

In this paper we examined the initial responses of LIP neurons to flashed stimuli. Most neurons with visual activity had a rapid, precisely timed on-response that was often followed by a brief period of inactivity. These data show that LIP receives a temporally precise message signaling the onset of a new stimulus; this information could be used to direct attention rapidly toward the new stimulus.

Latencies for the ring stimuli ranged from 34 to $60 \mathrm{msec}$ (mean, $41.4 \mathrm{msec}$ ) and increased to $42-76 \mathrm{msec}$ (mean, 49.5 $\mathrm{msec}$ ) when the target was presented in the RF. These latencies are generally shorter than those reported originally for the posterior parietal cortex as a whole (Bushnell et al., 1981) or for LIP (Barash et al., 1991). There are several possible reasons for this difference, such as stimulus size, contrast, duration, location in RF, bin size ( 2 msec compared with $20 \mathrm{msec}$ ), and the method used to confirm stimulus onset. In addition, it is possible that because we had only two target and four probe locations the animals learned to anticipate the stimulus onsets, and the resultant "expectancy" shortened latency. However, this is very unlikely to be the case, for several reasons. First, the stimulus timings and locations were randomized from trial to trial. Second, on some trials no target or distractor appeared in the RF. In these trials we found no response at the time that the target or distractor might have been expected to appear in RF (J.W. Bisley and M.E. Goldberg, unpublished observations). Finally, the latencies to the probe and distractor at the locus of attention (where the monkey was presumably expecting the stimulus) were no different from the latencies to these stimuli elsewhere.

Bair et al. (2002) have shown onset latencies ranging from 30 to $50 \mathrm{msec}$ in area $\mathrm{MT}$, an area receiving information directly from V1 and projecting to LIP. The middle superior temporal (MST) area is another direct recipient of MT projections (like LIP), and Kawano et al. (1994) found that MST latencies range from 37 to $86 \mathrm{msec}$, with a mean of $47.1 \mathrm{msec}$. The range and distribution of these data are very similar to those shown in Figure 2. Thus, the latencies measured in the current study are consistent with previous results and highlight the speed with which information can pass through the visual system.

We show here that prior attention allocation has no effect on the magnitude or latency of the initial burst. Attention factors similarly have no effect on the onset response in lower areas, such as MT, V2, and V4 (Treue, 2001). The only substantial factor found to affect latency was stimulus type: latencies to the rings were shorter than latencies to the probes, which were in turn shorter than latencies to the target or distractor. Such differences related to stimulus characteristics could simply mirror similar differences observed in the input areas (Reich et al., 2001). In most neurons the response to the task-irrelevant distractor was characterized by an initial burst followed by a brief pause in activity, and then often followed by a more sustained visual response. It was only this later activity that was affected by prior attention allocation. The lack of influence of attention factors on latency and response magnitude, combined with the fact that the latencies are extremely short and only slightly longer than those in area MT, strongly suggests that the initial burst is a pure visual response that travels quickly through the visual system. We speculate that it arises from input through a rapid V1-MT-LIP connection (Andersen et al., 1990) in the magnocellular pathway.

Although the actual function of LIP is still a matter of dispute (Dickinson et al., 2003), most studies agree that it has a complicated, cognitive function that transcends simple visual analysis (Andersen and Buneo, 2003; Bisley and Goldberg, 2003b). The activity of neurons in LIP has been related to decision making (Roitman and Shadlen, 2002), reward value (Platt and Glimcher, 1999), time keeping (Leon and Shadlen, 2003), saccade planning (Snyder et al., 1998), and salience (Gottlieb et al., 1998). We found that only the later sustained visual activity, emerging $\sim 40-50$ msec after the beginning of the response, reflects these more cognitive functions. The pause in visual activity between the onset burst and the later sustained visual response could represent a period during which the purely sensory response that travels quickly through the visual system has ended, but the longer latency visual response that is subject to cognitive modulation has not yet occurred. It is also possible that the pause represents a period of inhibition brought about by neighboring LIP neurons; however, the lack of a clear relationship between the initial visual response and the presence or absence of inactivity suggests that this may not be the case.

In addition to the short latencies, we found that most neurons had little variation in latency across trials. This, coupled with high firing rates during the burst, produced highly reproducible first spike times. These responses were as precise as those seen to stimulus changes in the LGN (Reinagel and Reid, 2000) and in area MT (Buracas et al., 1998); adding to the growing body of literature indicating that timing precision is not diminished at successive stages of visual processing. A coincidence detector could look at the precisely timed burst of activity from these neurons and detect the timing of stimulus onset with high precision (Lisman, 1997). If the activity across LIP is used to allocate attention, this would allow a quick and reliable orienting of attention to suddenly appearing stimuli, a trait that would be evolutionarily advantageous.

\section{References}

Andersen RA, Buneo CA (2003) Sensorimotor integration in posterior parietal cortex. In: The parietal lobes (Siegel AM, Andersen RA, Freund H-J, Spencer DD, eds), pp 159-177. Philadelphia: Lippincott Williams and Wilkins.

Andersen RA, Asanuma C, Essick G, Siegel RM (1990) Corticocortical connections of anatomically and physiologically defined subdivisions within the inferior parietal lobule. J Comp Neurol 296:65-113.

Bair W, Cavanaugh JR, Smith MA, Movshon JA (2002) The timing of response onset and offset in macaque visual neurons. J Neurosci 22:3189-3205.

Barash S, Bracewell RM, Fogassi L, Gnadt JW, Andersen RA (1991) Saccaderelated activity in the lateral intraparietal area. I. Temporal properties; comparison with area 7a. J Neurophysiol 66:1095-1108.

Bisley JW, Goldberg ME (2003a) Neuronal activity in the lateral intraparietal area and spatial attention. Science 299:81-86.

Bisley JW, Goldberg ME (2003b) The role of the parietal cortex in the neural processing of saccadic eye movements. In: The parietal lobes (Siegel AM, Andersen RA, Freund H-J, Spencer DD, eds), pp 141-157. Philadelphia: Lippincott Williams and Wilkins.

Brivanlou IH, Warland DK, Meister M (1998) Mechanisms of concerted firing among retinal ganglion cells. Neuron 20:527-539.

Buracas GT, Zador AM, DeWeese MR, Albright TD (1998) Efficient discrimination of temporal patterns by motion-sensitive neurons in primate visual cortex. Neuron 20:959-969.

Bushnell MC, Goldberg ME, Robinson DL (1981) Behavioral enhancement 
of visual responses in monkey cerebral cortex. I. Modulation in posterior parietal cortex related to selective visual attention. J Neurophysiol 46:755-772.

Dickinson AR, Calton JL, Snyder LH (2003) Non-spatial saccade-specific activation in area LIP of monkey parietal cortex. J Neurophysiol 90:2460-2464.

Egeth HE, Yantis S (1997) Visual attention: control, representation, and time course. Annu Rev Psychol 48:269-297.

Gottlieb JP, Kusunoki M, Goldberg ME (1998) The representation of visual salience in monkey parietal cortex. Nature 391:481-484.

Hays AV, Richmond BJ, Optican LM (1982) A UNIX-based multiple process system for real-time data acquisition and control. WESCON Conf Proc 2:1-10.

Hoffman JE, Subramaniam B (1995) The role of visual attention in saccadic eye movements. Percept Psychophys 57:787-795.

Kawano K, Shidara M, Watanabe Y, Yamane S (1994) Neural activity in cortical area MST of alert monkey during ocular following responses. J Neurophysiol 71:2305-2324.

Leon MI, Shadlen MN (2003) Representation of time by neurons in the posterior parietal cortex of the macaque. Neuron 38:317-327.

Lisman JE (1997) Bursts as a unit of neural information: making unreliable synapses reliable. Trends Neurosci 20:38-43.

Liu RC, Tzonev S, Rebrik S, Miller KD (2001) Variability and information in a neural code of the cat lateral geniculate nucleus. J Neurophysiol $86: 2789-2806$.
Maunsell JH, Gibson JR (1992) Visual response latencies in striate cortex of the macaque monkey. J Neurophysiol 68:1332-1344.

Platt ML, Glimcher PW (1999) Neural correlates of decision variables in parietal cortex. Nature 400:233-238.

Powell KD, Goldberg ME (2000) Response of neurons in the lateral intraparietal area to a distractor flashed during the delay period of a memoryguided saccade. J Neurophysiol 84:301-310.

Reich DS, Mechler F, Victor JD (2001) Temporal coding of contrast in primary visual cortex: when, what, and why. J Neurophysiol 85:1039-1050.

Reinagel P, Reid RC (2000) Temporal coding of visual information in the thalamus. J Neurosci 20:5392-5400.

Roitman JD, Shadlen MN (2002) Response of neurons in the lateral intraparietal area during a combined visual discrimination reaction time task. J Neurosci 22:9475-9489.

Shepherd M, Findlay JM, Hockey RJ (1986) The relationship between eye movements and spatial attention. Q J Exp Psychol 38:475-491.

Snyder LH, Batista AP, Andersen RA (1998) Change in motor plan, without a change in the spatial locus of attention, modulates activity in posterior parietal cortex. J Neurophysiol 79:2814-2819.

Treue S (2001) Neural correlates of attention in primate visual cortex. Trends Neurosci 24:295-300.

Victor JD, Purpura KP (1996) Nature and precision of temporal coding in visual cortex: a metric-space analysis. J Neurophysiol 76:1310-1326.

Yantis S, Jonides J (1984) Abrupt visual onsets and selective attention: evidence from visual search. J Exp Psychol Hum Percept Perform 10:601-621. 